lips and of fingers. From his replies to questions he seemed a man of emotional nature. I made another darkroom examination and again failed to find anything abnormal there. I made up my mind as to diagnosis (i.e., functional blindness). To make sure, I called him again next morning and prescribed in the meantime a blue pill to be taken at night and followed up by mag. sulph. in the morning. He went back with his relatives to the chemist to get the medicine. On the way the young man had a violent sneeze and immediately recovered his sight. Next morning he entered my room unaided, which confirmed my diagnosis of functional blindness. To make matters doubly sure, I sent him to a well-known physician for examination. The physician did not find anything organically wrong with him except that he had a few bad teeth which required looking after. All his organs were sound and healthy. Urine, normal. Reflexes, exaggerated. I think, it would be well if I here quote the physician's own sentence. "He (the patient) has told me all his history and I conclude that he is a case of hysteria.

The treatment needs no special comment."

During my practice of 20 years, this is the first case of its kind that I have seen.

Yours truly,

S. P. SHROFF.

P.S.-Field of vision in both eyes slightly contracted concentrically. DELH .

April 13, 1928.

\title{
THE TREATMENT OF HYPOPYON ULCER
}

\section{To the Editor of The British Journal of Ophthalmology.}

SIR,-Since reading the various plans of treatment for hypopyon ulcer, I would like to state my method of dealing with such cases.

Following upon the introduction of the colloidal compounds of mercury and silver (Crookes), I have practically used no other remedies in the past eight to ten years. I practically never use cautery or carbolic acid. As soon as a case is recognised-and the earlier the better-it is admitted to hospital, if a poor patient, and treatment with colloidal mercury is started.

Now it is useless to instil it three or four times a day. What is 
done is to instil it every hour, or if a very bad case, every half-hour, day and night, for the first 24 hours. The nurse is given definite instructions what to do. A stream of 10-20 drops is put well into the conjunctiva. The nurse waits one minute, timing herself, and the instillation is repeated, and again repeated, three instillations being given. In this way full effect of the drops is obtained. The patient must be wakened up to have the drops : this is essential.

The next 24 hours it is instilled about every $1 \frac{1}{2}$ hours, and the third day every two hours. This rarely fails to check the spread of the ulcer.

Atropine is used once or twice daily to control iritis, and the patient invariably expresses relief from pain and irritation.

If there is a dirty tear sac, the lower canaliculus must be slit well up into the sac, as the late Dr. Argyll Robertson used to do, and the sac frequently pressed out, and the discharge washed away with collosol hydrarg. In my opinion the results are remarkable. In proof of this I may quote the following. A successful cataract operation at the end of $\mathbf{1 0}$ days developed a septic ulcer in the site of the incision with pus dropping down into the anterior chamber, causing me great alarm. The above plan of treatment checked it entirely. A month ago a workman developed a bad septic ulcer on the lower half of the cornea. Collosol hydrarg checked it entirely and healed it.

The great advantage of this plan of treatment is that it is simple and painless, and douching with large quantities of lotion not necessary. At the end of a few days the colloidal mercury begins to irritate, then colloidal silver can be used or a simple lotion. The colloidal preparations must be fresh and not have been exposed to bright light for a long period.

In all cases the condition of the tear sac must be noted, also the condition of the nose and mouth. A dirty mouth, bad teeth, pyorrhoea, accumulations of tartar, etc., are a frequent cause of eye disease and some infections of the eye.

I have tried compound colourings but have not found them satisfactory. Compound colourings consist of scarlet red, methylene blue, gentian violet made up in the form of an ointment. It is successful in a number of cases, but one of the difficulties is to get it well retained in the eye between the lids.

In my opinion no plan of treatment equals the very frequent application of fresh colloidal mercury to the ulcer.

Yours truly,

G. Victor MiLLER

STOCKTON-ON-TEES. 